\title{
Operators and Products in the Lattice of Existence Varieties of Regular Semigroups
}

\author{
Norman R. Reilly and Shuhua Zhang \\ Communicated by F. Pastijn
}

When this article was published in Semigroup Forum [1], the title was inadvertently truncated to the first line only. The error was the fault of the Journal Secretary, who regrets the ommission of the second line of the title.

\section{References}

[1] Reilly, Norman R. and Shuhua Zhang, Operators and Products in the Lattice of Existence Varieties of Regular Semigroups, , Semigroup Forum 53 (1996), $1-24$. 